'Programa de formación de especialidad en Obstetricia y Ginecología, Facultad de Medicina, Pontificia Universidad Católica de Chile, Santiago, Chile 2Unidad de Medicina Materno-Fetal

División de Obstetricia y Ginecología

Facultad de Medicina Pontificia Universidad Católica de Chile, Santiago,

Recibido el 14 de abril de 2013, aceptado el 22 de agosto de 2013.

Correspondencia a: María Fernanda Garrido Oyarzún

Lira 85, División de Obstetricia y ginecología. E-mail: garrido.fda@gmail.

\section{Síndrome de HELLP normotensivo: caso clínico}

\author{
MARÍA FERNANDA GARRIDO ${ }^{1}$, JORGE ANDRÉS CARVAJAL ${ }^{2, a}$
}

HELLP syndrome (hemolysis, elevated liver enzymes and low platelets) represents a severe form of preeclampsia associated with serious maternal and fetal morbidities and even death. Usually HELLP syndrome appears during the evolution of a severe preeclampsia; however HELLP syndrome can have atypical clinical manifestations. We report a 22-year-old female who consulted at 20 weeks of pregnancy for lumbar pain, dysuria and fever. A urinalysis revealed the presence of white blood cells. The patient was admitted to hospital and treated with antimicrobials. Forty eight hours after admission a proteinuria of $440 \mathrm{mg} / 24 \mathrm{~h}$ was detected. A severe pre eclampsiaHELLP syndrome was diagnosed. However the patient remained with normal blood pressure. An elevation of liver enzymes and creatinine were observed. Five days later fetal death was confirmed and an abortion was induced. The patient required a platelet transfusion. Four days after the abortion, the patient was discharged in good conditions.

(Rev Med Chile 2013; 141: 1470-1474)

Key words: HELLP syndrome; Hypertension pregnancy-induced; Preeclampsia.

L a preeclampsia $(\mathrm{PE})$ es una patología multisistémica del embarazo, caracterizada por aparición de hipertensión y proteinuria. La fisiopatología de la PE no ha sido completamente esclarecida, pero se postula que se origina en una placentación anómala y la liberación de factores que causan disfunción endotelial. El síndrome de HELLP es una forma severa de preeclampsia (PE) que afecta a 0,2 a $0,6 \%$ de los embarazos ${ }^{1,2}$.

El diagnóstico de $\mathrm{PE}$ se formula en pacientes embarazadas con PAS $\geq 140 \mathrm{mmHg}$ o $\mathrm{PAD} \geq$ $90 \mathrm{mmHg}$ más proteinuria $\geq 300 \mathrm{mg} / 24 \mathrm{~h}$. Los criterios diagnósticos para el síndrome de HELLP son: anemia hemolítica microangiopática (esquistocitos en frotis y LDH > $600 \mathrm{UI} / \mathrm{L}$ ), elevación de transaminasas (AST $>70 \mathrm{UI} / \mathrm{L}$ ) y trombocitopenia (plaquetas $\left.<100.000 \mathrm{~mm}^{3}\right)^{3}$.

En raras ocasiones el HELLP puede tener presentaciones atípicas: inicio antes de las 20 semanas de embarazo, ausencia de proteinuria o hipertensión, e inicio o exacerbación postparto ${ }^{2,3}$.
Presentamos una mujer que desarrolla un síndrome de HELLP a las 21 semanas de embarazo, cuya presión arterial (PA) permanece en rango normal durante toda su evolución.

\section{Caso clínico}

Primigesta, 22 años, sin antecedentes mórbidos, controlada desde las 7 semanas; examen físico normal, PA 110/70 mmHg; exámenes prenatales normales y ecografía compatible con 7 semanas de embarazo, acorde con la fecha de última menstruación. Mantuvo controles regulares a las 11, 15 y 19 semanas de embarazo, manteniendo PA en rango 100-110/60-70 $\mathrm{mmHg}$.

Consultó con $20+5$ semanas de embarazo, por dolor lumbar bilateral sordo, de $4 \mathrm{~h}$ de evolución, asociado a disuria y sensación febril.

Al ingreso se detectó PA 130/85 $\mathrm{mmHg}$, frecuencia cardiaca 86 por min y temperatura 
$37,8{ }^{\circ} \mathrm{C}$. Examen físico normal, excepto puño percusión positiva bilateral. Sedimento de orina mostraba leucocitos 15-20 y bacterias ++ , hematocrito $35,8 \%$, leucocitos $13.800 \mathrm{~mm}^{3}$ sin desviación izquierda, plaquetas $70.400 \mathrm{~mm}^{3}$; PCR $12 \mathrm{mg} / \mathrm{dl}$, amilasa, función renal y pruebas de coagulación normales.

Se hospitalizó con diagnóstico de pielonefritis aguda e inició tratamiento antibiótico endovenoso con clindamicina y gentamicina. La paciente evolucionó afebril, normotensa (120/80), refiriendo dolor moderado en hemiabdomen superior, sin hallazgos al examen físico.

Luego de $24 \mathrm{~h}$ desde el ingreso, los exámenes mostraron hematocrito $32,9 \%$ con esquistocitos al frotis, plaquetas $59.100 \mathrm{~mm}^{3}$, GPT $402 \mathrm{U} / \mathrm{L}$, GOT $32 \mathrm{U} / \mathrm{L}$, ácido úrico $4,8 \mathrm{mg} / \mathrm{dl}$, fosfatasas alcalinas (FA) 108 U/L. Ecografía de abdomen y pelvis normal. Ecografía obstétrica: feto único, líquido amniótico normal, placenta normoinserta, biometría concordante con 201/2 semanas; en la evaluación anatómica destaca una fisura labiopalatina y el resto normal. Doppler de arteria umbilical con latidos cardiacos fetales (LCF) en 140 por min, flujo diastólico ausente intermitente (índice pulsatilidad (IP) 1,62); ducto venoso mostraba aumento de resistencia (IP 1,49, onda a presente) y arterias uterinas con escotadura bilateral y aumento de resistencia (IP promedio 2,65).

A las $48 \mathrm{~h}$ desde el ingreso, los análisis fueron: urocultivo negativo (se suspendió tratamiento antibiótico), proteinuria $440 \mathrm{mg} / 24 \mathrm{~h}$, clearance de creatinina $120 \mathrm{ml} / \mathrm{min}$. Con estos antecedentes se postula el diagnóstico de preeclampsia severa (PES) - HELLP, destacando la persistencia de PA normal (120/80).

La paciente evolucionó normotensa (Figura 1), con remisión del dolor abdominal, reflejos osteotendineos normales y pruebas de laboratorio con disminución paulatina del hematocrito y plaquetas, asociado a aumento progresivo de las enzimas hepáticas y elevación de la creatinina (Tabla 1). Dada la evolución del laboratorio, cursando un embarazo de $21+2$ semanas se confirmó el diagnóstico de síndrome de HELLP. Se decidió manejo conservador con vigilancia materna para intentar alcanzar la viabilidad fetal y se planificó el inicio de dexametasona (la que finalmente no se administró). Sin embargo, al quinto día se constató ausencia de LCF (muerte fetal in útero). Se decidió inducción del aborto con misoprostol, bajo infusión continua de sulfato de magnesio ( 5 g carga y $1 \mathrm{~g} / \mathrm{h}$ mantención por $24 \mathrm{~h}$ post aborto). Se obtuvo un feto muerto de sexo masculino, 310 gramos, fisura labio-palatina izquierda, sin otras malformaciones evidentes. Placenta $260 \mathrm{~g}$, aspecto normal. Se realizó revisión instrumental en pabellón. Por recuento plaquetario en 32.600/ $\mathrm{mm}^{3}$ se transfundió $6 \mathrm{U}$ de plaquetas con recuento de control de $53.500 / \mathrm{mm}^{3}$.

La paciente evolucionó en buenas condiciones generales, asintomática, siempre normotensa (Figura 1), con mejoría de los parámetros de laboratorio (Tabla 1). Fue dada de alta al cuarto día post aborto.

En control ambulatorio a los 11 días de puerperio, se encontró normotensa (PA 108/74), proteinuria de $24 \mathrm{~h}$ negativa. La biopsia placentaria informó extensa zona de infarto hemorrágico (30\% de la masa total).

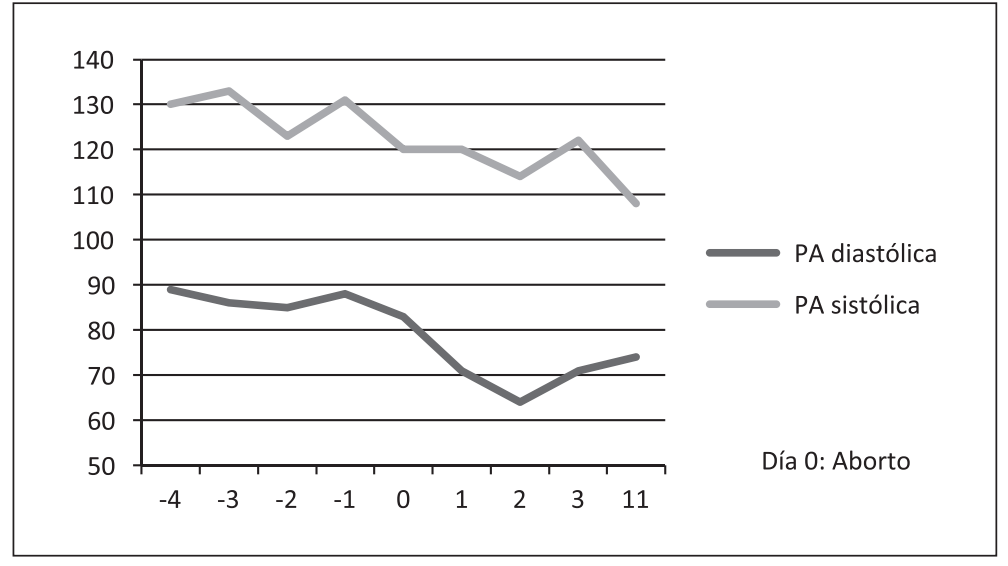

Figura 1. Curva de presión arterial. Embarazada de 21 semanas que desarrolla un síndrome de HELLP, pero que mantiene PA normal durante toda la evolución del cuadro. 
Tabla 1. Pruebas de laboratorio previo y posterior a la interrupción del embarazo

\begin{tabular}{|c|c|c|c|c|c|c|c|c|c|c|c|c|c|}
\hline Días & Hora & Hto & Plaq & LDH & ALT & AST & FA & $\mathbf{A U}$ & Bili & $\mathrm{Cr}$ & BUN & TP & TTPA \\
\hline-4 & $0: 27$ & 35,8 & 70,4 & & & & & & & 0,45 & 7 & & \\
\hline-4 & 7:06 & 32,9 & 59,1 & & & & & & & & & & \\
\hline-3 & & 32,3 & 34,0 & & 402 & 32,6 & & & 0,46 & & 9 & & \\
\hline-2 & $17: 31$ & 30 & 60,8 & 259 & 168 & 57 & 108 & 4,8 & 0,21 & 0,55 & & & \\
\hline-1 & & 32,3 & 66,3 & 298 & 131 & 46 & 109 & 5,3 & & 0,63 & & 11,5 & 33 \\
\hline-1 & $20: 27$ & 32,6 & 71,0 & 306 & 134 & 74 & 107 & 4,7 & & 0,56 & 11 & 11,7 & 38 \\
\hline 0 & $2: 22$ & 33,4 & 64,2 & 368 & 156 & 117 & & 4,4 & & 0,6 & 10 & 11,9 & 38 \\
\hline 0 & $7: 43$ & 33,3 & 47,4 & 530 & 193 & 212 & 117 & 4,6 & & 0,61 & 10 & 12,1 & 39 \\
\hline 0 & $13: 20$ & 34,8 & 32,6 & 1.199 & 342 & 520 & 133 & 5,4 & 0,75 & 0,66 & & 13,8 & 41 \\
\hline+1 & $1: 10$ & 27,2 & 37,5 & 1.991 & 589 & 1.042 & 1.199 & 5,6 & 0,75 & 0,77 & 17 & 14,4 & 38 \\
\hline+1 & $8: 22$ & 26,6 & 39,7 & 2.032 & 761 & 1.208 & 130 & & 0,59 & 0,69 & 17 & & \\
\hline+1 & $12: 16$ & 30,9 & 48,8 & 1.414 & 774 & 1.145 & 149 & & 0,46 & 0,66 & 14 & 13,6 & 42 \\
\hline+1 & $18: 16$ & 29,1 & 53,8 & 854 & 701 & 754 & 145 & 5,0 & 0,41 & 0,69 & 14 & 12,1 & 37 \\
\hline+2 & 8:00 & 30,8 & 87,8 & 497 & 514 & 328 & 140 & 4,7 & 0,26 & 0,6 & & 12,8 & 32 \\
\hline+3 & & 27,1 & 152 & 284 & 220 & 44 & 109 & 5,3 & & 0,64 & 10 & 14 & 35 \\
\hline+11 & & 33,8 & 161 & 236 & 29 & 32 & 94 & & 0,34 & 0,7 & 8 & & \\
\hline
\end{tabular}

Días (-): Días previos a interrupción del embarazo; 0: Interrupción del embarazo; Días (+): Días posteriores a la interrupción del embarazo; Hto: hematocrito; Plaq: plaquetas; LDH: lactato deshidrogenasa; ALT: alaninoaminotranferasa; AST: aspartato aminotransferasa; FA: fostatas alcalinas; AU: Ácido úrico; Bili: bilirrubina total; Cr: creatinina plasmática; BUN: nitrógeno ureico; TP: tiempo de protrombina; TTPA: tiempo de trombina parcial activado.

\section{Discusión}

Comunicamos el caso de una mujer de 22 años quien desarrolla en su primer embarazo un síndrome de HELLP con PA en rango normal durante toda la evolución de la enfermedad.

El HELLP es una complicación grave de la PE y presente en aproximadamente $20 \%$ de las mujeres con PES ${ }^{4}$. La PES se asocia a una tasa de mortalidad y morbilidad materna de $0,2 \%$ y $5 \%$ respectivamente ${ }^{4}$.

Las mujeres que desarrollan PES y HELLP tienen 3 a 25 veces mayor riesgo de complicaciones graves como coagulación intravascular diseminada (15\%), falla renal (3\%), edema pulmonar (8\%), hemorragia intracraneana $(1,5 \%)$, requerimiento de transfusión sanguínea (25\%) y desprendimiento prematuro de placenta normoinserta $(4,1-22,9 \%)$ que un embarazo no complicado 5 . A largo plazo, aproximadamente $20 \%$ desarrollará hipertensión o microalbuminuria, además de aumento del riesgo de morir por enfermedad cardiovascular, especialmente en aquellas con PES de inicio precoz ${ }^{6}$. Desde el punto de vista fetal, la mortalidad perinatal de la PES varía entre 3,9 a $16,6 \%$ entre las 24 a 34 semanas y 71 a $100 \%$ antes de las 25 semanas manejados en forma expectante, mientras que el HELLP reporta una mortalidad perinatal de 22,6\% (5-8). Se estima que el diagnóstico oportuno de HELLP permitiría evitar estas complicaciones.

Este caso ilustra las dificultades diagnósticas que se producen frente a las presentaciones atípicas de la PE y HELLP. La paciente consultó por dolor lumbar bilateral y presentó un alza térmica que desvió inicialmente el estudio hacia un cuadro de infección urinaria alta, sin embargo, evolucionó afebril, asintomática y con urocultivo negativo descartándose una pielonefritis aguda. Mantuvo PA $<140 / 90 \mathrm{mmHg}$ durante toda su evolución, desarrollando un HELLP oligosintomático y a una edad gestacional precoz. El diagnóstico se basó en 
proteinuria de $24 \mathrm{~h}>300 \mathrm{mg}$, elevación progresiva de las transaminasas, anemia con signos de hemólisis y caída progresiva del recuento plaquetario.

Posterior al aborto, presenta recuperación rápida de los parámetros de laboratorio, incluyendo LDH, plaquetas y transaminasas, evolución característica de un HELLP posterior a la interrupción del embarazo, siendo esto un aspecto clave en la certificación del diagnóstico ${ }^{9}$. Cabe destacar que aunque la PA no sobrepasó los 140/90 mmHg, la paciente presenta una caída en la curva de PA significativa los primeros días de puerperio, que se hace más evidente al control de los 11 días.

Evidencia reciente sugiere que en algunas pacientes, la PE se manifestará en forma de aumento de la permeabilidad capilar, con proteinuria, ascitis y edema; o como un amplio espectro de alteraciones hemostáticas con afección multiorgánica. Estas pacientes presentarán alteraciones de los parámetros de laboratorio compatibles con HELLP y proteinuria, pero no desarrollarán hipertensión arterial $^{3}$.

Los diagnósticos diferenciales a considerar son los denominados imitadores de PES: hígado graso agudo del embarazo, púrpura trombocitopénico trombótico, síndrome hemolítico urémico y crisis de lupus eritematoso sistémico (LES).

El hígado graso agudo del embarazo es una complicación del embarazo que afecta a 1 en 10.000 a 15.000 partos, más frecuente en primigestas y embarazos múltiples, durante el tercer trimestre del embarazo. Presenta síntomas y signos que se superponen con el HELLP. En el caso presentado, el dolor abdominal, proteinuria, elevación de transaminasas y trombocitopenia en aumento hasta la interrupción del embarazo y la mejoría tras 48-72 h apoyan ambos diagnósticos. Sin embargo, dos elementos característicos del hígado graso agudo no se manifestaron; elevación de la bilirrubina (presente en 100\% de los casos) y ausencia de anemia ${ }^{9}$. Nuestra paciente evolucionó con bilirrubinemia normal y anemia leve, por lo que se descartó esta patología.

El púrpura trombocitopénico trombótico (PTT) y el síndrome hemolítico urémico (SHU) son microangiopatías trombóticas extremadamente infrecuentes durante el embarazo ${ }^{10}$. El PTT al igual que el HELLP, presenta trombocitopenia, anemia hemolítica microangiopática y elevación de las transaminasas, todas presentes en nuestra paciente. Sin embargo, no manifestó alteraciones neurológicas, hiperbilirrubinemia ni hematuria características del PTT, por lo que se descartó este diagnóstico.

Los pocos casos de SHU reportados en el embarazo han sido en el postparto tardío. La proteinuria, edema, anemia hemolítica y elevación de las transaminasas presentes en la paciente apoyaban tanto el diagnóstico de SHU como el de HELLP. No obstante, no conformaba el grupo de riesgo epidemiológico ni presentó compromiso de la función renal (habitualmente grave en el SHU) ${ }^{10}$, descartándose el diagnóstico.

Una exacerbación lúpica con nefritis, al igual que el HELLP, se presenta con anemia hemolítica microangiopática y trombocitopenia ${ }^{10}$, como nuestra paciente. Sin embargo, no tuvo alteración de la función renal, fiebre persistente, artralgias ni lesiones cutáneas características de una exacerbación lúpica, por lo que fue descartada, si bien no se realizó estudio de autoanticuerpos ${ }^{10}$.

Es difícil diferenciar estos cuadros clínicos cuando no están presentes otros síntomas o signos característicos de PE. Sin embargo, con la interrupción del embarazo, la paciente tuvo rápida reversión de los parámetros de laboratorio lo que confirma un HELLP, ya que el PTT, el SHU y la exacerbación lúpica no mejoran con el parto/aborto.

Conocemos tres casos reportados de HELLP normotensivo en embarazos simples. Este es el primero diagnosticado en el segundo trimestre del embarazo en una mujer normotensa.

Basados en nuestra experiencia, recomendamos mantener un alto índice de sospecha en pacientes con síntomas, signos y hallazgos de laboratorio sugerentes de preeclampsia o HELLP aún en ausencia de hipertensión o a edades gestacionales precoces, ya que la preeclampsia severa y el HELLP pueden tener presentaciones atípicas. El diagnóstico e interrupción oportuna del embarazo en el caso de un HELLP debe realizarse independiente de la edad gestacional, evitando otras complicaciones graves, incluso la muerte materna.

\section{Referencias}

1. Weinstein L. Syndrome of hemolysis, elevated liver enzymes, and low platelet count: a severe consequence of hypertension in pregnancy. Am J Obstet Gynecol 1982; 142 (2): 159-67. 
2. Stella CL, Malik KM, Sibai BM. 2008. HELLP syndrome: an atypical presentation. Am J Obstet Gynecol 2008; 198 (5): e6-8.

3. Stella CL, Sibai BM. Preeclampsia: Diagnosis and management of the atypical presentation. J Matern Fetal Neonatal Med 2006; 19 (7): 381-6.

4. ACOG Committee on Practice Bulletins-Obstetrics. Diagnosis and management of preeclampsia and eclampsia Number 33. Obstet Gynecol 2002; 99 (1): 159-67.

5. Sibai BM, Barton JR. Expectant management of severe preeclampsia remote from term: patient selection, treatment, and delivery indications. Am J Obstet Gynecol 2007; 196 (6): 514.e1-9.

6. Ghulmiyyah L, Sibai B. Maternal mortality from preeclampsia/eclampsia. Semin Perinatol 2012; 36 (1): 56-9.
7. Belghiti J, Kayem G, Tsatsaris V, Goffinet F, Sibai BM, Haddad B. Benefits and risks of expectant management of severe preeclampsia at less than 26 weeks gestation: the impact of gestational age and severe fetal growth restriction. Am J Obstet Gynecol 2011; 205 (5): 465 e16.

8. Reubinoff BE, Schenker JG. HELLP syndrome-a syndrome of hemolysis, elevated liver enzymes and low platelet count-complicating preeclampsia-eclampsia. Int J Gynaecol Obstet 1991; 36 (2): 95.

9. Martin JN Jr, Blake PG, Perry KG Jr, McCaul JF, Hess LW, Martin RW. The natural history of HELLP syndrome: patterns of disease progression and regression. Am J Obstet Gynecol 1991; 164 (6 Pt 1): 1500.

10. Sibai MD. Imitators of severe pre-eclampsia. Semin Perinatol 2009. 33 (3): 196-205. 